\title{
Optimum Table Spacing in Predistorting Amplifier Linearizers
}

\author{
James K. Cavers
}

\begin{abstract}
Radio frequency (RF) power amplifiers require linearization to reduce the intermodulation (IM) power if the signal does not have a constant envelope. Digital predistortion (PD) is one of the more successful methods. However, implementations usually employ uniform spacing of the PD lookup table entries, since the question of optimum nonuniform spacing has been unresolved. This paper provides the solution: a way to compute the IM for different spacing methods, a comparison of several table spacing schemes, a derivation of the optimum table spacing method, and a demonstration that the IM of optimum spacing is only a few decibels lower than that of equal spacing by amplitude when variations in operating point are considered.
\end{abstract}

Index Terms-Linearization, power amplifiers, radio transceivers.

\section{INTRODUCTION}

A RADIO frequency (RF) power amplifier (PA) is a nonlinear device. When it carries a signal that does not have a constant envelope, such as linear modulation, a group of carriers or the sum of several code-division multiple-access (CDMA) signals, the PA generates intermodulation (IM) distortion. Since the IM power falls into adjacent channels as interference, the design of linearizers has become a key technology in modem mobile and personal communication transceivers.

A powerful type of linearizer is the digital predistorter (PD) [1]-[5], which incorporates a lookup table (LUT) containing a representation of the inverse characteristic of the amplifier. The cascade of PD and PA is almost perfectly linear. Because it is not tied to a low-order parametric model (such as a polynomial) and is not sensitive to loop delay, the digital PD provides excellent IM suppression.

Reported work has the entries equispaced in power [1], [2], [4], because of its computational simplicity, or equispaced in amplitude [3], [5], because it frequently provides greater IM reduction. Most authors [1], [3]-[5] also note that nonuniform spacing, in which table entries are more closely spaced where the amplifier characteristics vary sharply, would provide better performance. Beyond this observation, however, there has been no progress to date on nonuniform spacing.

This paper provides answers in nonuniform spacing. It demonstrates how to calculate the IM power for arbitrary spacing, by introducing a companding function into table

Manuscript received January 18, 1996; revised June 21, 1998. This work was supported by Glenayre R\&D, Inc., Vancouver, Canada.

The author is with the School of Engineering Science, Simon Fraser University, Burnaby, B.C., V5A IS6, Canada (e-mail: cavers@sfu-ca).

Publisher Item Identifier S 0018-9545(99)07398-3.

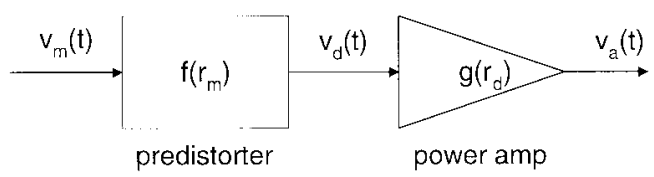

Fig. 1. Relation of predistorter to power amplifier.

indexing. It then obtains a simple expression for the optimum spacing of LUT entries for a given amplifier, modulation format, and number of table entries. Numerical results compare the reduction in IM power using optimized spacing with that achievable by simpler methods, including spacing that is uniform in amplitude, power and $\mu$-law amplitude. The results reported here are related to, and as basic as, the theory of optimum quantization [6].

\section{MODELS}

\section{A. Component Models}

Fig. 1 shows the predistorter and PA as a complex baseband model (in the actual circuit, the PA is an RF device, and there is a quadrature modulator between PD and PA). The complex envelope $v_{m}(t)$ of the modulation enters from a modem or analog speech processor, and the PD applies a memoryless nonlinearity to produce the predistorted signal $v_{d}(t)$ according to

$$
v_{d}=v_{m} f\left(r_{m}\right)
$$

where $r_{m}(t)=\left|v_{m}(t)\right|$ is the amplitude of the modulation and $f(r)$ is the complex gain of the predistorter, which summarizes $\mathrm{AM} / \mathrm{AM}$ and AM/PM effects. The PA is modeled similarly, with the amplifier output $v_{a}(t)$ given by

$$
v_{a}=v_{d} g\left(r_{d}\right)
$$

where $r_{d}(t)=\left|v_{d}(t)\right|$ is the amplitude of the predistorted signal and $g(r)$ is the complex gain of the amplifier. For convenience in the analysis, we assume the amplifier to be normalized so that saturation occurs at unit amplitude of its input and results in unit output amplitude; equivalently, $|g(1)|=1$. Fig. 2 shows a typical class $\mathrm{AB}$ amplifier characteristic, with the transfer characteristic (amplitude out versus amplitude in) and the magnitude and phase of the complex gain.

Ideally, the cascade of PD and PA produces a simple linear gain $K$, so that $v_{a}(t)=K v_{m}(t)$. Substitution of (1) into (2) shows that the condition to be satisfied by the optimum PD 


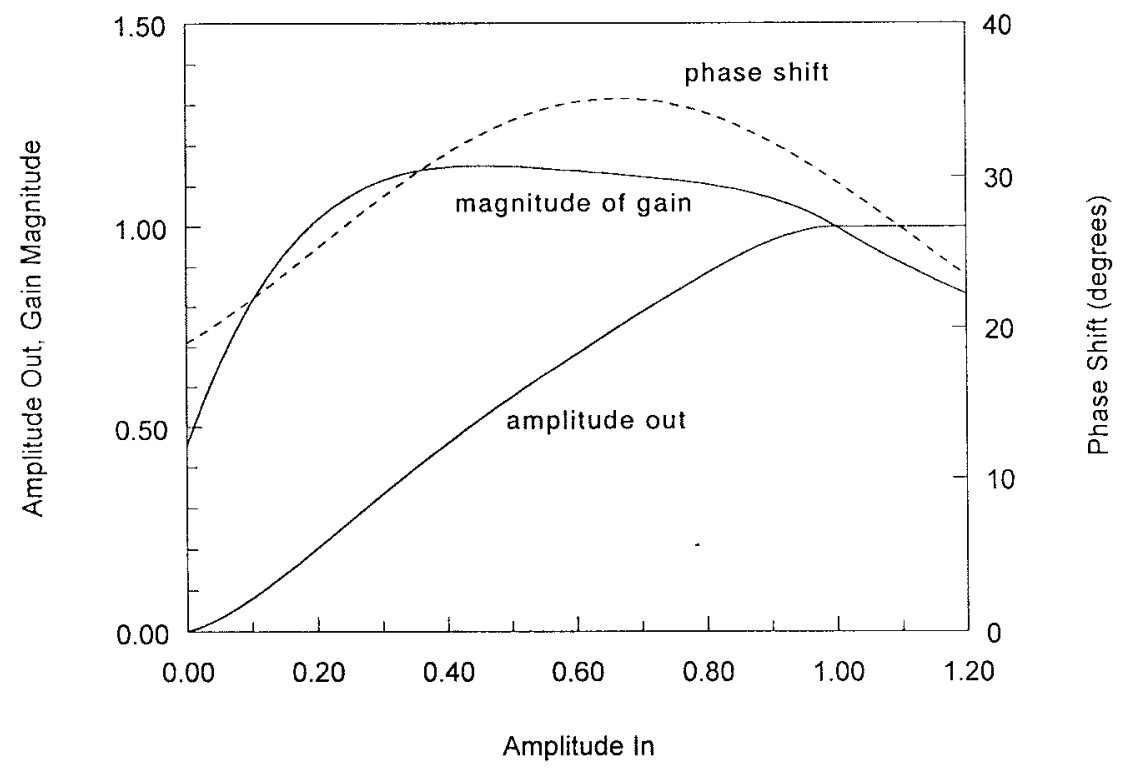

Fig. 2. Characteristics of the Class $\mathrm{AB}$ amplifier.

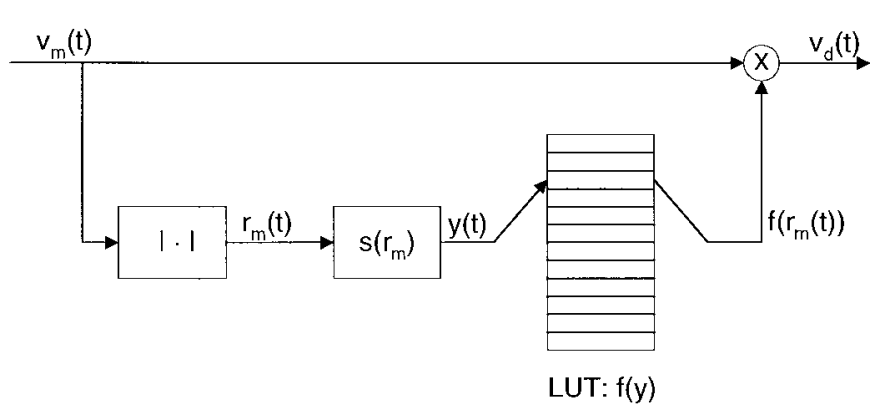

Fig. 3. Internal structure of the predistorter.

complex gain, denoted $f_{o}\left(r_{m}\right)$, is

$$
f_{o}\left(r_{m}\right) g_{o}\left(r_{m}\right)=K
$$

where

$$
g_{o}\left(r_{m}\right)=g\left(r_{m}\left|f_{o}\left(r_{m}\right)\right|\right)
$$

is defined for notational convenience.

The derivatives of the complex gains $f^{\prime}(r)=d f(r) / d r$ and $g^{\prime}(r)=d g(r) / d r$ are important. Because it occurs frequently, we also define

$$
g_{o}^{\prime}\left(r_{m}\right)=g^{\prime}\left(r_{m}\left|f_{o}\left(r_{m}\right)\right|\right) .
$$

Note that $g_{o}^{\prime}(r) \neq d g_{o}(r) / d r$.

\section{B. Structure of Predistorter}

As shown in Fig. 3, the PD implements (1) using a LUT with entries equispaced in $y$, an index variable directly related to the amplitude $r_{m}$. No interpolation is assumed in this analysis, so the resulting complex gain is piecewise constant. We assume that adaptation algorithms [1]-[5] make each table entry optimum in the sense of (3) at the midpoint of its range.

The key to analysis of nonuniform spacing is the companding function $s(r)$ inserted between the calculation of amplitude $r_{m}$ and the index variable $y=s\left(r_{m}\right)$. Because the amplifier model is normalized to saturate at unit amplitude, it is convenient to define this function over the same interval. Consequently, $s(0)=0$ and $s(1)=1$, and it is monotonically increasing, so that $s^{\prime}(r)>0$ for $0 \leq r \leq 1$. The simplest example is indexing the table by amplitude [3], [5], where $s(r)=r$. Indexing by power, as in [1], [2], and [4], corresponds to $s(r)=r^{2}$.

Regions of $r$ where table entries are relatively dense correspond to regions where $s^{\prime}(r)$ is relatively large. If the table contains $N_{t}$ entries, the width of the bins (i.e., the spacing) in the $y$ domain is $1 / N_{r}$. To first order, the corresponding width of bins in the $r_{m}$ domain is

$$
d=1 \frac{1}{N_{t} s^{\prime}\left(r_{m}\right)}
$$

\section{Signal PDF and Input Backoff}

Because of the nonlinear characteristic, IM power depends upon the input backoff (IBO), defined as the ratio of actual input power to input power required for saturation (the latter value is unity because of amplifier normalization) and upon the pdf of the signal amplitude. We will model the signal amplitude statistics using a pdf with a mean square value of IBO and maximum value of one, to represent clipping at saturation. This analysis ignores any distortion due to clipping, since no single-amplifier linearization method can compensate for it. Because power control and backoff are included in the amplitude pdf, we can assume that the ideal linear gain $K=1$, without loss of generality. Thus, even after linearization, unit input amplitude results in unit output amplitude and saturation.

Although the results of this paper apply to any modulation format, we will use a specific probability density function for purposes of illustration. It represents multicarrier operation, in which the number of independent frequency-division multipleaccess (FDMA) or CDMA carriers is sufficient that the their sum can be approximated as Gaussian. In this case, the 


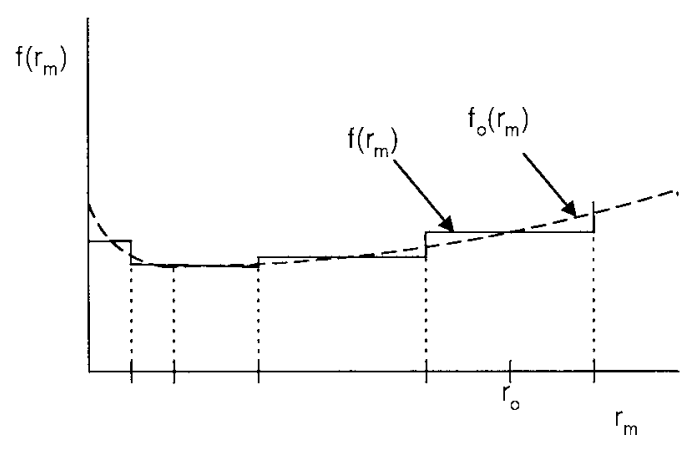

Fig. 4. Optimum and quantized predistortion function.

amplitude has a truncated Rayleigh pdf

$$
\begin{gathered}
P_{r}(r)=\frac{2 r}{\mathrm{IBO}} \exp \left(-\frac{r^{2}}{\mathrm{IBO}}\right)\left(1-\exp \left(-\frac{1}{\mathrm{IBO}}\right)\right)^{-1}, \\
0 \leq r \leq 1 .
\end{gathered}
$$

\section{DistORTION ANALYSIS}

\section{A. Residual IM Power}

Because the PD is defined only at table points, its complex gain is slightly in error at other input values, producing an error

$$
v_{a e}=v_{a}-K v_{m}
$$

in the PA output. If $f=f_{o}+\delta f$ is the perturbed complex gain, then from (1) to (4) the output error is

$$
\begin{aligned}
v_{a e} & =v_{m}\left(f_{o}+\delta f\right) g\left(r_{m}\left|f_{o}+\delta f\right|\right)-K v_{m} \\
& \approx v_{m}\left(\delta f g_{o}+f_{o} g_{o}^{\prime} r_{m} \operatorname{Re}\left[\frac{f_{o}^{*}}{\left|f_{o}\right|} \delta f\right]\right)
\end{aligned}
$$

where the approximation is to first order in $\delta f$. Next, consider a single bin in the $r_{m}$ domain, as shown in Fig. 4, and assume that its table entry is optimized at the midpoint $r_{o}$ of the bin, i.e., its value is $f_{o}\left(r_{o}\right)$. At nearby amplitudes $r_{m}=r_{o}+\delta r$ in the bin, the piecewise constant table has a gain error

$$
\delta f=-f_{o}^{\prime}\left(r_{o}\right) \delta r
$$

Substitution into (9) gives the output error as

$$
v_{a e}=v_{m}\left(-f_{o}^{\prime} g_{o}-\frac{f_{o}}{\left|f_{o}\right|} g_{o}^{\prime} r_{m} \operatorname{Re}\left[f_{o}^{*} f_{o}^{\prime}\right]\right) \delta r
$$

We can relate the derivatives $f_{\circ}^{\prime}$ and $g_{\circ}^{\prime}$ by substituting (4) into (3) and differentiating, to obtain

$$
g_{o} f_{o}^{\prime}+f_{o} g_{o}^{\prime}\left(\left|f_{o}\right|+r_{m} \operatorname{Re}\left[f_{o}^{\prime} \frac{f_{o}^{*}}{\left|f_{o}\right|}\right]\right)=0 .
$$

Substituting (12) into (11), then using (3) and the convention that $K=1$, we have

$$
v_{a e}=v_{m} \frac{g_{o}^{\prime}}{g_{o}\left|g_{o}\right|} \delta r
$$

Next, for small bins we can expect that $\delta r$ is uniformly distributed across the bin with zero mean, and that $v_{m}, g_{o}$, and $g_{o}^{\prime}$ do not change appreciably. In this case, the IM power contributed by the bin is the expectation

$$
x_{a e}=E\left[\left|v_{a e}\right|^{2}\right]=r_{m}^{2} \frac{\left|g_{o}^{\prime}\right|^{2}}{\left|g_{o}\right|^{4}} \frac{d^{2}}{12}
$$

where $d$ is the width of the bin.

The development in (8) to (14) parallels that in [1], where the amplifier characteristic is represented as a function of instantaneous power, instead of amplitude. It is also related to [5], where trigonometric relations are employed instead.

We link (14) with the effect of the compander by substituting (6) to obtain the IM as a function of input level. Finally, we take the expectation over the signal amplitude pdf to obtain the total output power as

$$
P_{a e}=\int_{0}^{1} \frac{w\left(r_{m}\right)}{s^{\prime}\left(r_{m}\right)^{2}} d r_{m}
$$

where $w\left(r_{m}\right)$ is the nonnegative function

$$
w\left(r_{m}\right)=\frac{r_{m}^{2}}{12 N_{t}^{2}} \frac{\left|g_{o}^{\prime}\left(r_{m}\right)\right|^{2}}{\left|g_{o}\left(r_{m}\right)\right|^{4}} p_{r}\left(r_{m}\right) .
$$

By means of (15), we can calculate the IM power resulting from any companding function $s(r)$.

\section{B. Optimized Spacing}

What is the best spacing of PD table entries? Equivalently, what is the best companding function? To find out, we minimize (15) subject to the constraints $s(0)=0, s(1)=1$, and $s^{\prime}\left(r_{m}\right)>0$ for $0 \leq r_{m} \leq 1$. Using a Lagrange multiplier to represent the constraint, we minimize

$$
\int_{0}^{1} \frac{w\left(r_{m}\right)}{s^{\prime}\left(r_{m}\right)^{2}} d r_{m}+\lambda\left(\int_{0}^{1} s^{\prime}\left(r_{m}\right) d r_{m}-1\right)
$$

Setting the gradient with respect to $s^{\prime}\left(r_{m}\right)$ to zero, we have the optimum function

$$
s_{o}^{\prime}\left(r_{m}\right)=\left(\frac{2 w\left(r_{m}\right)}{\lambda}\right)^{1 / 3} .
$$

Substituting this function into the constraint, we have

$$
\lambda=\left(\int_{0}^{1}\left(2 w\left(r_{m}\right)\right)^{1 / 3} d r_{m}\right)^{3}
$$

and

$$
s_{o}^{\prime}\left(r_{m}\right)=\left(\int_{0}^{1} w\left(r_{m}\right)^{1 / 3} d r_{m}\right)^{-1} w\left(r_{m}\right)^{1 / 3} .
$$

Note that the optimum companding function, unlike fixed functions such as amplitude, power or $\mu$-law companding, depends on both the amplifier and the signal pdf and backoff.

What is the performance of optimum spacing? Substitute (20) into (15) to obtain the lowest possible IM power for a given amplifier, modulation, backoff, and number of table entries

$$
P_{a e o}=\left(\int_{0}^{1} w\left(r_{m}\right)^{1 / 3} d r_{m}\right)^{3}
$$




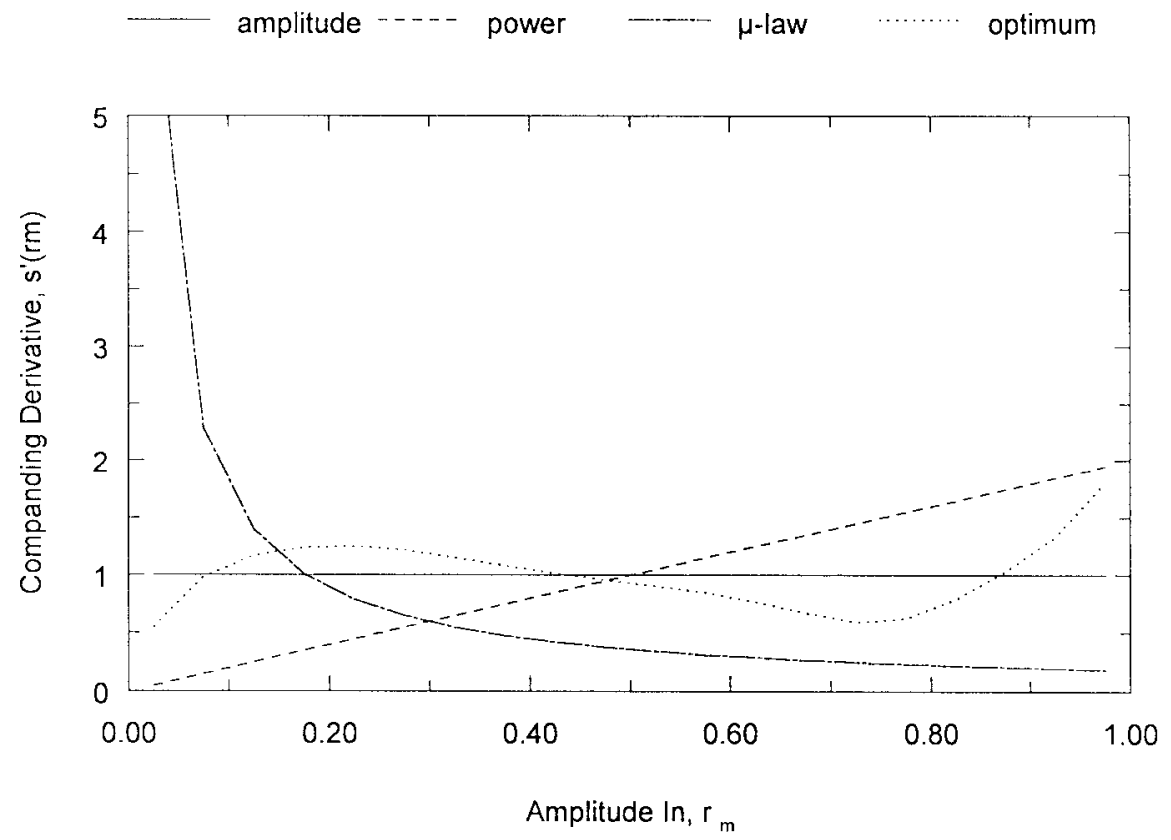

Fig. 5. Derivatives of companding functions.

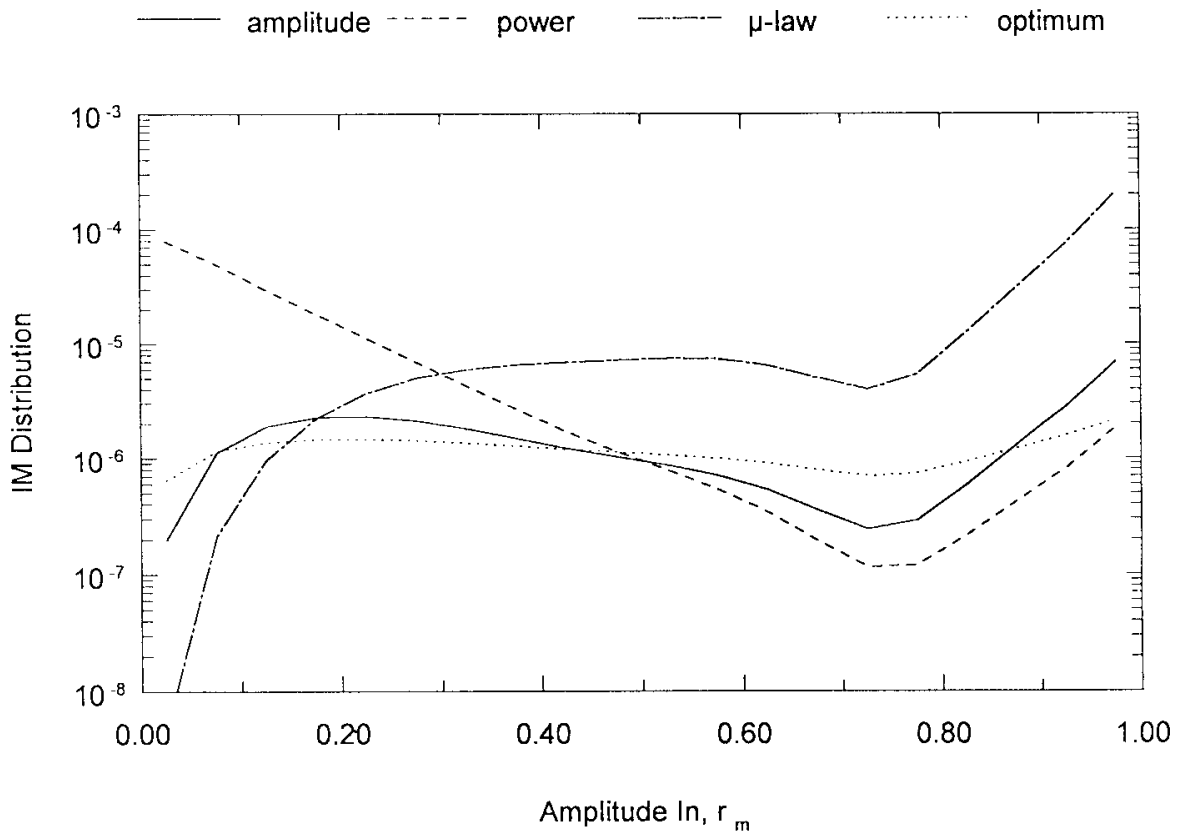

Fig. 6. Distribution of IM power by amplitude at 3-dB backoff.

\section{Performance of Selected Companding Functions}

In this section, we compare the IM resulting from four different companding functions. The first two are for equal spacing in amplitude and in power, respectively

$$
\begin{array}{llrl}
s_{a}\left(r_{m}\right)=r_{m} & s_{a}^{\prime}\left(r_{m}\right)=1 & \text { (amplitude) } \\
s_{p}\left(r_{m}\right)=r_{m}^{2} & s_{p}^{\prime}\left(r_{m}\right)=2 r_{m} & \text { (power). }
\end{array}
$$

Clearly, equispacing by power makes entries denser near saturation, at the expense of the cutoff region. This would not be unreasonable if one were linearizing a Class A amplifier to obtain more power efficiency. However, it is more likely that Classes $\mathrm{AB}$ or $\mathrm{B}$ amplifiers would be employed, and we shall see below that equispacing by power has significant disadvantages, especially in a system with power control where large backoff values might be used. For the third function, we try $\mu$-law amplitude, in the hope that it provides a more constant signal to IM ratio as the backoff is varied

$$
\begin{aligned}
& s_{\mu}\left(r_{m}\right)=\frac{\ln \left(1+\mu r_{m}\right)}{\ln (1+\mu)} \\
& s_{\mu}^{\prime}\left(r_{m}\right)=\frac{\mu}{\ln (1+\mu)} \frac{1}{1+\mu r_{m}} \quad(\mu \text { law }) .
\end{aligned}
$$




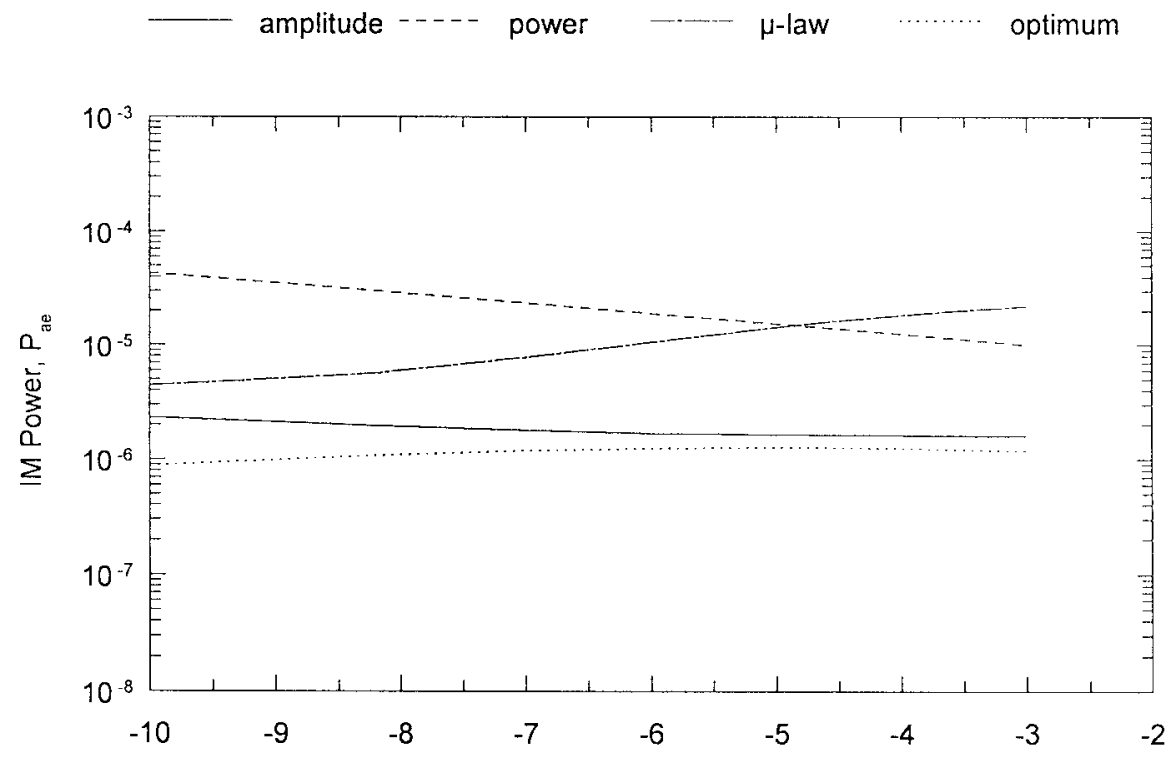

IBO, dB

Fig. 7. Total IM power as a function of input backoff.

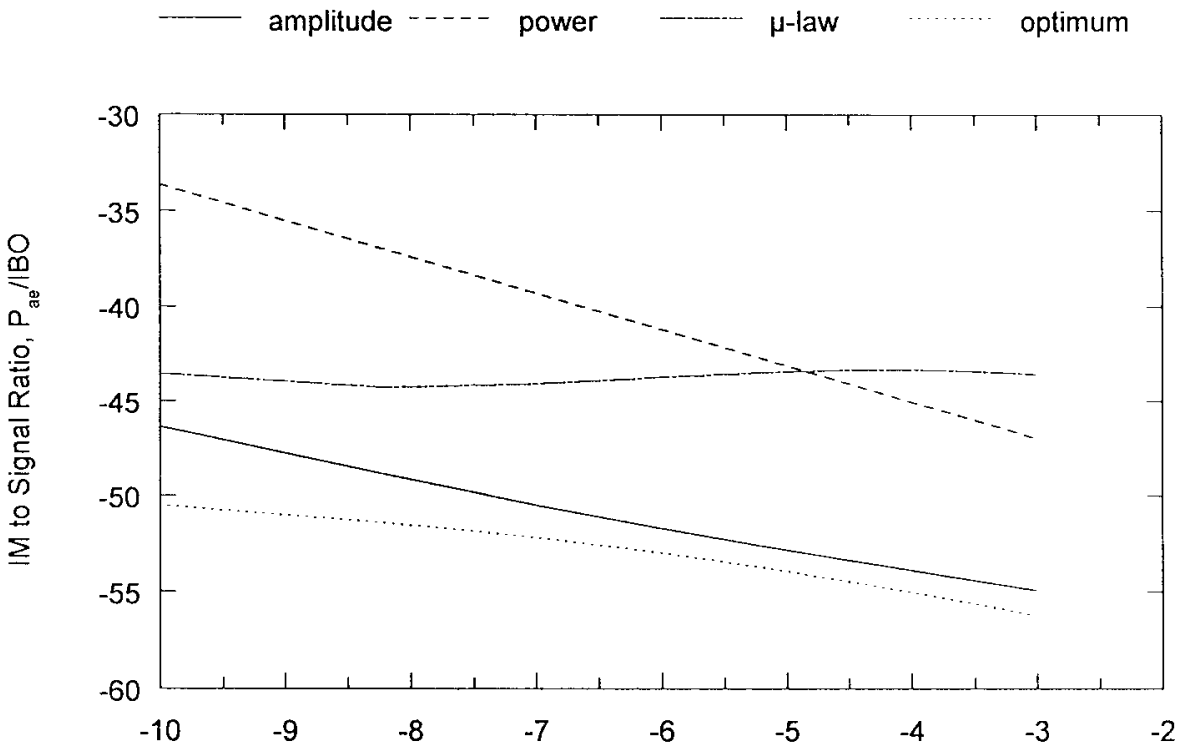

$18 \mathrm{~B}, \mathrm{~dB}$

Fig. 8. IM to signal power ratio as a function of input backoff.

Their performance is obtained from (15). The fourth companding function is the optimum function (20), with performance given by (21) if it is optimized at every IBO value. We will also examine (20), optimized at a specific IBO value but used at all backoffs; in this case, we again use (15) to calculate IM power.

The first point of interest is the density, or reciprocal spacing, of table entries. From (6), the density is proportional to the derivative of the companding function. Fig. 5 shows that optimum spacing provides a balance between the saturation and cutoff regions. In contrast, spacing by power makes entries dense near saturation, and $\mu$ law concentrates entries at low- amplitude levels. Also of interest is the way the resulting IM power is distributed in amplitude. This quantity is given by the integrand in (15) and is shown in Fig. 6 for a table of 64 entries and a backoff of $3 \mathrm{~dB}$. It can be seen that optimum indexing results in the most constant distribution of IM power, followed by equispacing in amplitude. In contrast, spacing by power and by $\mu$ law suffer from significant IM power generation at low levels and high levels, respectively, because their table entries are unnecessarily concentrated at the other end of the amplitude range. The total IM power $P_{a e}$, shown in Fig. 7 as a function of backoff IBO, follows in a predictable way from these comments; power spacing, for example, produces more 


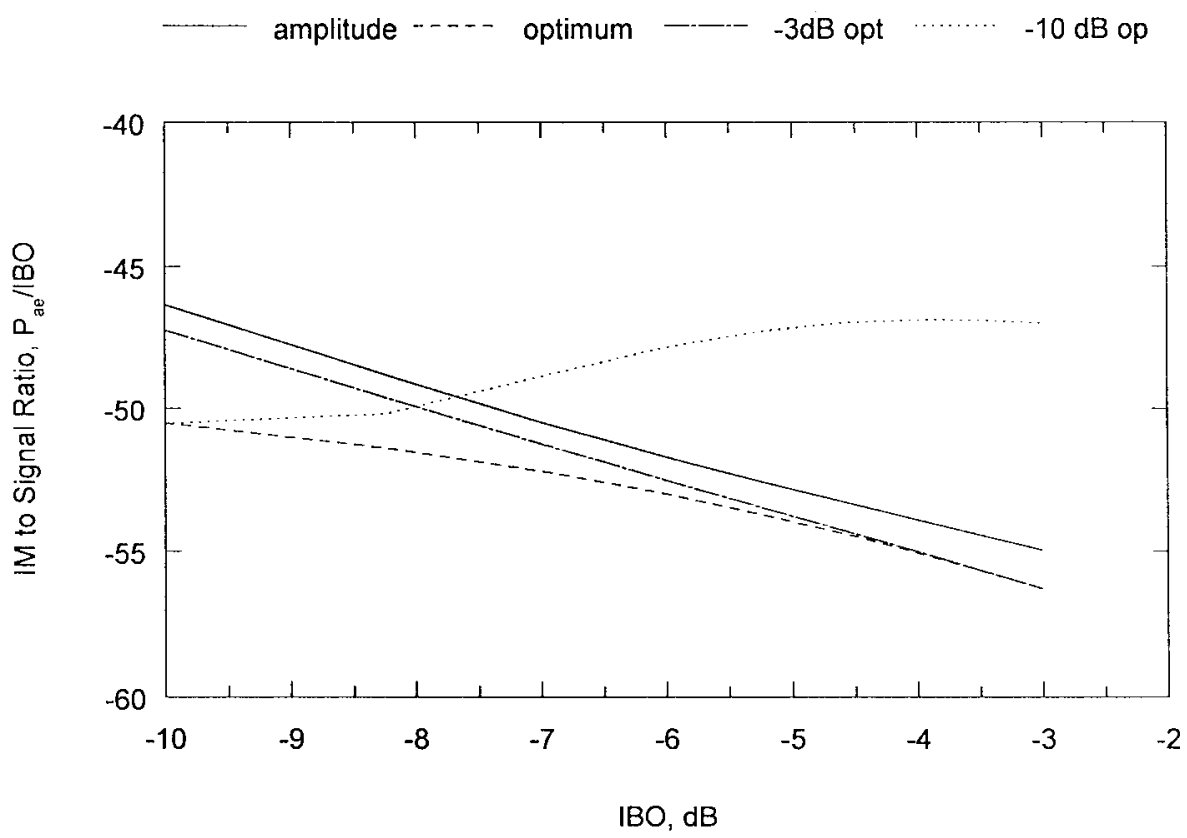

Fig. 9. IM to signal power ratio using companding functions optimized for $-3-$ and $-10-d B$ IBO.

IM power when the amplifier backoff increases, and $\mu$-law spacing produces less.

The primary quality measure is the ratio of IM power to signal power, given on a per-sample basis by $P_{a e} / \mathrm{IBO}$. The corresponding ratio of power spectra is given approximately by a further multiplication by the ratio of signal bandwidth to sampling rate, since the IM power is relatively white [1]. Fig. 8 shows the per-sample result for a table of 64 entries. As expected, equispacing in power degrades rapidly with greater backoff, for two reasons: the lower levels have greater distortion power, and the signal power itself is reduced. Also as expected, spacing by $\mu$ law gives relatively constant performance, since IM power and signal power increase and decrease together. Unfortunately, its performance, though considerably better than spacing by power at greater backoffs, is everywhere much poorer than spacing by amplitude, with a disparity of $10 \mathrm{~dB}$ at small to moderate backoffs. Optimum spacing produces between 1-4 dB less IM power than spacing by amplitude. We must remember, though, that the optimum companding function depends on amplitude and pdf, which means that a different function, or distribution of spacing, is required at every backoff value. This is certainly possible by means of a separate table storing the break points for every allowable backoff value, but it is cumbersome.

This introduces the question of mismatch between optimization conditions and operating conditions. In particular, we consider a companding function optimized for one particular backoff value, but used at all backoffs, simply as a matter of simplified design. Fig. 9 shows the result for optimization IBO values of 3 and $10 \mathrm{~dB}$. Clearly, it is preferable to optimize at the higher value. Equally clearly, the result provides only about 1-dB improvement compared to the simpler spacing by amplitude.

To assess how robust these results are to changes of amplifier or modulation, we repeated the calculations for the
BLU-98 transistor used in related studies [3], biased for Class $\mathrm{AB}$ operation, and for a pdf resembling that of QPSK with $35 \%$ root cosine shaping. Apart from changes in absolute level, the relative performance of the four table spacing methods was very similar to the results displayed here. When biased for Class B operation, the difference between spacing by amplitude and spacing optimized for the operating point increases by about $2 \mathrm{~dB}$, so that the gap is about $6 \mathrm{~dB}$ for large backoff and $3 \mathrm{~dB}$ for small backoff, and $\mu$-law spacing is roughly parallel to amplitude spacing. In other respects, the curves and the conclusions are similar to those for Class AB.

\section{CONCLUSIONS}

This paper has presented two new basic results: a systematic way to describe and analyze arbitrary nonuniform spacing of predistortion table entries; and simple expressions for the optimum nonuniform spacing and its performance. It also provided a numerical comparison of four candidate methods: equispacing by amplitude, power and $\mu$ law, all of which are fixed, and optimum spacing, which depends on amplifier and backoff.

We have seen that, for a Class $\mathrm{AB}$ amplifier, equispacing by power is by far the worst method almost everywhere, and that equispacing by $\mu$ law is least dependent on the changes in backoff level experienced in a system with dynamic power control. However, equispacing by amplitude produces less IM power than the other two fixed methods, by amounts ranging from 4 to $10 \mathrm{~dB}$. As for optimized spacing, it is from 1-4 $\mathrm{dB}$ better than equispacing by amplitude. Unfortunately, an operating scenario in which the spacing is optimized for one backoff value but used at all backoffs produces disappointing results-when optimized for small backoffs, it is only about $1 \mathrm{~dB}$ better than the simpler equispacing by amplitude, and when optimized for large backoffs, it is far 
worse than amplitude spacing over most of the range. We note finally that equispacing by power may still be appropriate for a Class A amplifier in a system without dynamic power control.

We conclude from this investigation that making predistortion tables equispaced in amplitude is an excellent choice from an engineering standpoint: it is simple, it does not depend on amplifier, modulation format or backoff, and its performance is very close to the limit defined by optimum spacing.

\section{REFERENCES}

[1] J. K. Cavers, "Amplifier linearization using a digital predistorter with fast adaptation and low memory requirements," IEEE Trans. Veh. Technol., vol. 39, pp. 374-382, Nov. 1990.

[2] A. Wright and W. Durtler, "Experimental performance of an adaptive digital linearized power amplifier," IEEE Trans. Veh. Technol., vol. 41, pp. 395-400, Nov. 1992

[3] M. Faulkner and M. Johannson, "Adaptive linearization using predistortion-Experimental results," IEEE Trans. Veh. Technol., vol. 43, pp. 323-332, May 1994.

[4] I. K. Lau, C. G. Englefield, and P. A. Goud, "Innovative DSP implementation of adaptive RF power amplifier linearizers," in Proc. Wireless'94, Calgary, Canada.
[5] L. Sundstrom, M. Faulkner, and M. Johansson, "Quantization analysis and design of a digital predistortion linearizer for RF power amplifiers," IEEE Trans. Veh. Technol., vol. 45, pp. 707-719, Nov. 1996.

[6] A. Gersho, "Principles of quantization," IEEE Trans. Circuits Syst., vol. CAS-25, no. 7, July 1978.

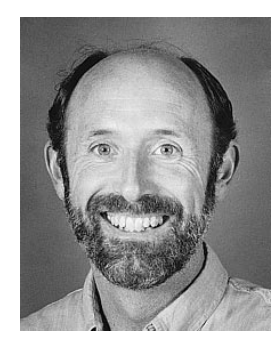

James K. Cavers was born in Port Alice, B.C., Canada, in 1944. He received the B.A.Sc. degree in engineering physics in 1966 and the Ph.D. degree in electrical engineering in 1970 , both from the University of British Columbia, Canada.

From 1970 to 1979, he was an Assistant and then Associate Professor in the Department of Systems Engineering, Carleton University, Ottawa, Canada. From 1979 to 1982, he was a Program Manager at MacDonald Dettwiler and Associates, Vancouver, Canada, followed by a year as a Senior Engineer at Glenayre Electronics, Vancouver. In 1983, he joined the School of Engineering Science, Simon Fraser University, Burnaby, B.C., where he holds the rank of Professor. From 1990 to 1994, he was the Director of the School of Engineering Science. His research interests include modulation and detection for mobile communications and integrated RF/DSP design.

Dr. Cavers is the 1992 recipient of the Stentor Telecommunications Research Award and the 1995 recipient of the Gold Medal in Engineering and Applied Science from the Science Council of B.C. 